\title{
A high dynamic radiation measurement instrument: the Bolometric Oscillation Sensor (BOS)
}

\author{
P. Zhu ${ }^{1}$, M. van Ruymbeke ${ }^{1}$, Ö. Karatekin ${ }^{1}$, J.-P. Noël ${ }^{1}$, G. Thuillier ${ }^{3}$, S. Dewitte ${ }^{2}$, A. Chevalier ${ }^{2}$, C. Conscience , $^{2}$ \\ E. Janssen ${ }^{2}$, M. Meftah ${ }^{3}$, and A. Irbah \\ ${ }^{1}$ Royal Observatory of Belgium, Av. Circulaire 3, B1180 Bruxelles, Belgium \\ ${ }^{2}$ Royal Meteorological Institute of Belgium, Av. Circulaire 3, B1180 Bruxelles, Belgium \\ ${ }^{3}$ LATMOS, 4 place Jussieu 75252 Paris CEDEX 05, France
}

Correspondence to: P. Zhu (zhuping@oma.be)

Received: 5 August 2014 - Published in Geosci. Instrum. Method. Data Syst. Discuss.: 3 December 2014

Revised: 1 April 2015 - Accepted: 24 April 2015 - Published: 8 May 2015

\begin{abstract}
The Bolometric Oscillation Sensor (BOS) is a broadband radiation measurement instrument onboard the PICARD satellite that was active between 2010 and 2014. The main detector is a thermistor attached black coated surface, which was permanently exposed to space without any optical and aperture accessories. The temperature measurements are used within a transfer function to determine variations in incoming solar irradiance as well as the terrestrial radiation. In the present article, the measurement principle of the BOS and its transfer function are presented. The performance of the instrument is discussed based on laboratory experiments and space observations from the PICARD satellite. The comparison of the short-term variation of total solar irradiance (TSI) with absolute radiometers such as VIRGO/SOHO and TIM/SORCE over the same period of time suggests that the BOS is a relatively much simpler but very effective sensor for monitoring electromagnetic radiation variations from visible to infrared wavelengths.
\end{abstract}

\section{Introduction}

The SOlar VAriability experiment for the PICARD mission (SOVAP) is one of the three instruments constituting the solar payload of CNES's PICARD microsatellite (Thullier et al., 2006). It is composed of a DIARAD-type absolute radiometer (Dewitte et al., 2004) and the Bolometric Oscillation Sensor (BOS) (van Ruymbeke et al., 2010, 2011), which measure the incoming radiation with sampling frequencies of 180 and $10 \mathrm{~s}$ respectively. The main requirement for the
BOS was to provide stable and precise measurements of the incoming electromagnetic radiation over the broad spectrum with a high $10 \mathrm{~s}$ cadence. The short-term solar irradiance variation from seconds to minutes remain less studied due to the nature of operation sequences of absolute radiometers. The sensing unit is usually operated in an opening and closing sequence. In order to reach the thermal radiation power equivalent, the exposer and closer time are dependent on the instrument structure itself. In general, one measurement takes at least $1 \mathrm{~min}$. The high-frequency measurement is strictly limited by it. In order to study some typical events, like the solar oscillation modes, the solar eclipses, the solar white flares, and the transit of other planets, high sampling data are preferred. The BOS sensor is developed to meet such requirements.

The absolute value of the solar irradiance was not requested a priori during the designing phase since the BOS was collocated to the DIARAD absolute radiometer, which is providing sound TSI measurement with rich space heritage (Mekaoui et al., 2010). The big challenge was rather to develop a small compact instrument that could continuously monitor the variation of the solar irradiance as well as of the terrestrial radiation with a high precision. To achieve this objective, the BOS team has developed an innovative design that enhances the performance of the instrument by reducing the electronic common mode and the ambient thermal noise.

The PICARD microsatellite with the BOS onboard was successfully launched on 15 June 2010. PICARD is a technological demonstration mission with a nominal duty cycle of 2 years. Following 2 years of operation, the mis- 

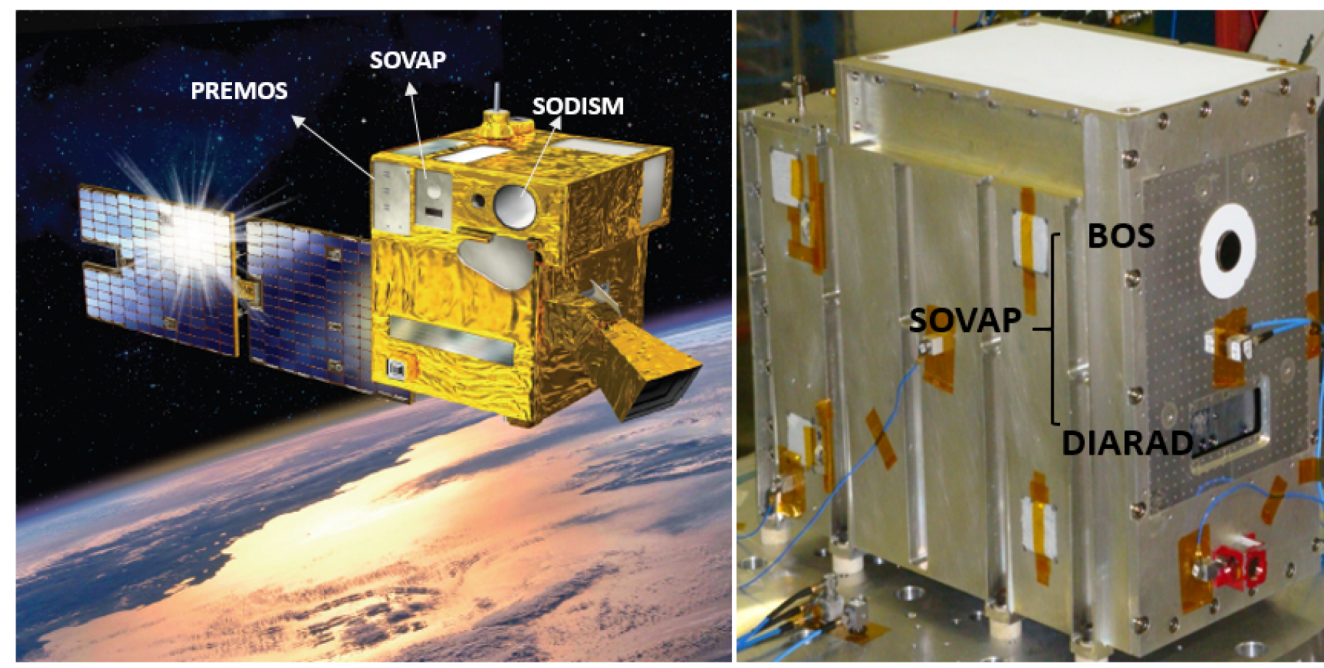

Figure 1. The PICARD satellite and SOVAP experiment. The BOS is integrated into the same unit with DIARAD (photo credits: CNES).
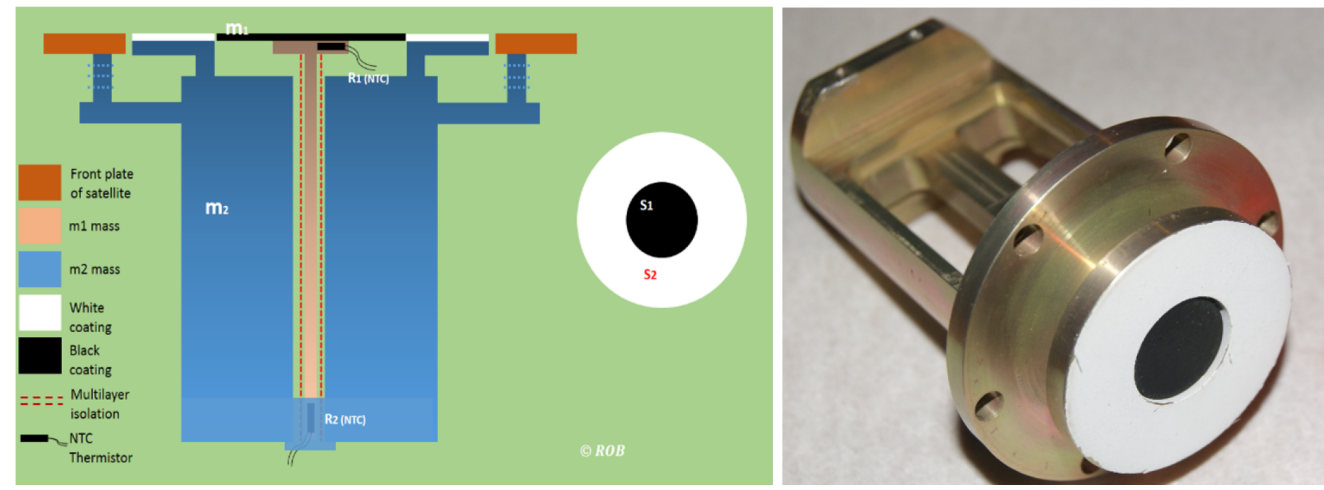

Figure 2. The sketch of the BOS. The detector $\left(m_{1}\right)$ is connected to the heat sink $\left(m_{2}\right)$ by a thin shunt, which is thermally isolated from $m_{2}$ by multi-layer isolation (MLI). The instrument unit is installed in the front panel of the spacecraft through a high thermal resistance Vespel ${ }^{\circledR}$ layer.

sion was extended for 1.5 years. PICARD has three experiments: SODISM (Meftah et al., 2014a) measures the diameter of the Sun, while PREMOS (Schmutz et al., 2013) and the SOVAP (Zhu et al., 2010; Meftah et al., 2014b) measure the total and spectral solar irradiance (Fig. 1). The BOS as a part of the SOVAP experiment has acquired data quasi-continuously over the 3.5 year mission duration. BOS data are archived and publically available on the website (http://picard-bos.oma.be).

In the next section the measurement principle of the BOS and its geometry are presented. Reduction of the electronic common mode and background ambient thermal noise is discussed in Sect. 3. The laboratory experiment and the flight performance of the instrument are respectively presented in Sects. 5 and 6. The space observation results are then compared with absolute radiometers VIRGO/SOHO and TIM/SORCE.

\section{Operating principle and geometry}

The measurement principle of the BOS is a combination of a thermistor-based bolometer and a pyrometer. The basic geometry is a cylinder where the heat flux measurement is realised through temperature measurements at thermal nodes (Fig. 2, left). The team has tested a number of prototypes before opting for this flying model (Fig. 2, right) (van Ruymbeke, 2006).

The sensing unit is composed of two pieces of mass: $m_{1}$ and $m_{2}$. It is made of aluminium, to which a special spacequalified treatment (Alodyne 1200) was applied before the final chemical coating. The surface of $m_{1}$ is painted in black and $m_{2}$ is painted in white with MAP ${ }^{\odot}$ coating. The mass $m_{1}$ is then connected to $m_{2}$ through a thin shunt at the bottom of $m_{2}$. It is thermally insulated from $m_{2}$ with multi-layer isolation. The length of the shunt was determined by computer simulations (Martinez, 2007). 
The working principle of the BOS is quite straightforward. The light mass $\left(m_{1}\right)$ absorbs and re-emits a broadband wavelength electromagnetic radiation from space. The heavier mass $\left(m_{2}\right)$ absorbs less energy and re-emits most energy caught by $m_{1}$ and $m_{2}$ back into space.

According to the thermal node analysis method, the thermal network of the BOS is approximated by the temperature at two diffusion nodes $T_{1}$ and $T_{2}$. Mathematically, the temperature of the diffusion node is modelled by the expression (K \& K Associates, 2000)

$\Sigma Q-C \frac{\mathrm{d} T}{\mathrm{~d} t}=0$,

with $\Sigma Q$ the sum of the thermal energy of the network, and $C$ the thermal capacitance. The instrument is thermally insulated from the outside with a high thermal resistance Vespe ${ }^{\circledR}$ layer. Supposing a constant temperature of the environment, the diffusion node can be approximated by

$\Sigma Q=\alpha s_{1} Q_{\text {in }}-\epsilon s_{1} \sigma T_{1}^{4}-K \Delta T$,

where $\alpha$ and $\epsilon$ are the absorption and emissivity coefficients of the black coating, $Q_{\text {in }}$ the incoming electromagnetic radiation, $s_{1}$ the surface area of mass $m_{1}, \sigma$ the Stefan-Boltzman coefficient, $K=k A / L$ the thermal conductance, $k$ the thermal conductivity of the shunt, $A$ and $L$ the surface area and length of the shunt, $\Delta T=T_{1}-T_{2}$ the difference between $T_{1}$ and the temperature measured under the black surface, and $T_{2}$ the temperature at the bottom end of the shunt (Fig. 2). The effect of the electronic common mode as well as the background ambient thermal noise are discussed in the next section.

From Eqs. (1) and (2), we can find a numerical solution of $Q_{\text {in }}$ with the values of two temperature measurements:

$Q_{\text {in }}=\frac{1}{\alpha s_{1}}\left(\epsilon s_{1} \sigma T_{1}^{4}+K \Delta T+C \frac{\mathrm{d} T_{1}}{\mathrm{~d} t}\right)$.

Since the sensing surface of $m_{1}$ is directly exposed to space with a hemispherical field of view (HFOV), it absorbs all electromagnetic radiation by principle. We use the Mini BetaCURVE polymide NTC (negative temperature coefficient) thermistor component as temperature detectors. These components were calibrated by measurement specialities. The thermistor resistance at $25^{\circ} \mathrm{C}$ is $100 \mathrm{~K} \Omega$. The tolerance of the beta value is $\pm 0.5 \%$ at $25^{\circ} \mathrm{C}$ (Measurements technical specialties ${ }^{\circledR}$ data sheet; Measurements specialties, 2005). Two electronic interfaces (EI) are separately connected to each thermistor. The V2F digital counting of each EI has a $120 \mathrm{~dB}$ dynamic bandwidth covering the $T_{1}$ working temperature range between -30 and $60^{\circ} \mathrm{C}$. The instrument is designed to be automatically off when the $T_{1}$ is out of this range. With such a large dynamic range, the resolution of the temperature measurement can theoretically reach $90 \mu^{\circ}$ $\left(90 \times 10^{-6}{ }^{\circ} \mathrm{C}\right)$.

\section{Reducing the electronic common mode and background ambient thermal noise}

The common mode is usually indicating the noise induced by the amplifier of electronics. Here, it is referred to as the electronic common mode (ECM).

In order to limit the overheating by the Joule effect, two resistances ( $74 \mathrm{~K} \Omega$ for $T_{1}$ and $82 \mathrm{~K} \Omega$ for $T_{2}$ ) are wired in series with NTC thermistors. The amplifier is separately applied to the output voltage of the first thermistor and to the voltage difference between the first and second thermistors. This configuration is essentially increasing the resolution of temperature difference measurements and reducing the ECM by a common electronic interface rather than individually counting the output of two NTC thermistors (van Ruymbeke et al., 2010).

The instrument package was installed at the front Sunpointing panel of the PICARD satellite and fixed with six screws. Each screw was surrounded by a layer of Vespel ${ }^{\circledR}$, which has a very low thermal conductivity coefficient $\left(0.005 \mathrm{~W} \mathrm{~cm}^{-1} \mathrm{~K}^{-1}\right)$ (Vespel technical information (Miller, 2000)). The mass ratio between $m_{2}$ and the main sensing unit $m_{1}$ is as high as 200. In addition, the energy absorbed by the black coated surface $s_{1}$ is 2 times higher than the white painted surface $m_{2}$ due to its strong reflectivity to visible radiation (Zhu et al., 2012). All these factors guarantee that the heat flux direction is from the main detector $m_{1}$ along the isolated shunt towards the junction between the shunt and $m_{2}$ during the Sun observation nominal phase. The surrounding ambient thermal noise is damped by the heat sink $\left(m_{2}\right)$. The temperature measured at $T_{2}$ has been slowly changing over the mission and can be considered to be a stable reference for short-period thermal modulation.

\section{Laboratory thermal vacuum test}

The laboratory experiments were conducted with the spare flight model (FM) of the BOS. The complete instrument was installed inside a vacuum tank. Since the model is connected to the inner wall of the vacuum tank through a support, the measurements are sensitive to the temperature of the environment due to the thermal conductivity between the supporting mass of the BOS and the vacuum tank. Therefore, it is important to do the experiments in a temperaturecontrolled room. The experiments are realised in a thermally controlled room designed originally for astronomy photo disk digitising. A climatisation installation delivered by Becker Reinraumtechnik ${ }^{\circledR}$ regulates the clean room to a constant air temperature of $20 \pm 0.05^{\circ} \mathrm{C}$ and to a relative air humidity of $50 \pm 1 \%$ (De Cuyper, personal communication). A Labsphere Uniform Source Integrating Sphere is selected as a radiation source (Labsphere PB-13019-000; Labsphere, 2005). The inner sphere of Labsphere is coated with Labsphere's highly reflective and durable Spectraflect ${ }^{\circledR}$ coating; 
Table 1. Laboratory experiment results.

\begin{tabular}{ccccccccc}
\hline Experiment & $T_{1}(\mathrm{~K})$ & $1 \sigma$ & $T_{2}(\mathrm{~K})$ & $1 \sigma$ & $T_{1}-T_{2}(\mathrm{~K})$ & $1 \sigma$ & $F\left(\mathrm{Wm}^{-2}\right)$ & $1 \sigma$ \\
\hline 1 & 294.888 & 0.005 & 294.469 & 0.004 & 0.423 & 0.001 & 83.6 & 0.08 \\
2 & 294.906 & 0.004 & 294.482 & 0.005 & 0.423 & 0.001 & 83.9 & 0.09 \\
3 & 294.904 & 0.010 & 294.487 & 0.007 & 0.423 & 0.002 & 83.9 & 0.10 \\
4 & 294.909 & 0.008 & 294.494 & 0.003 & 0.423 & 0.001 & 84.0 & 0.10 \\
5 & 294.884 & 0.010 & 294.473 & 0.003 & 0.423 & 0.002 & 83.7 & 0.09 \\
6 & 294.880 & 0.009 & 294.465 & 0.005 & 0.423 & 0.001 & 83.8 & 0.10 \\
7 & 294.891 & 0.007 & 294.471 & 0.006 & 0.423 & 0.001 & 83.9 & 0.09 \\
8 & 294.911 & 0.006 & 294.495 & 0.002 & 0.424 & 0.001 & 84.0 & 0.10 \\
\hline
\end{tabular}
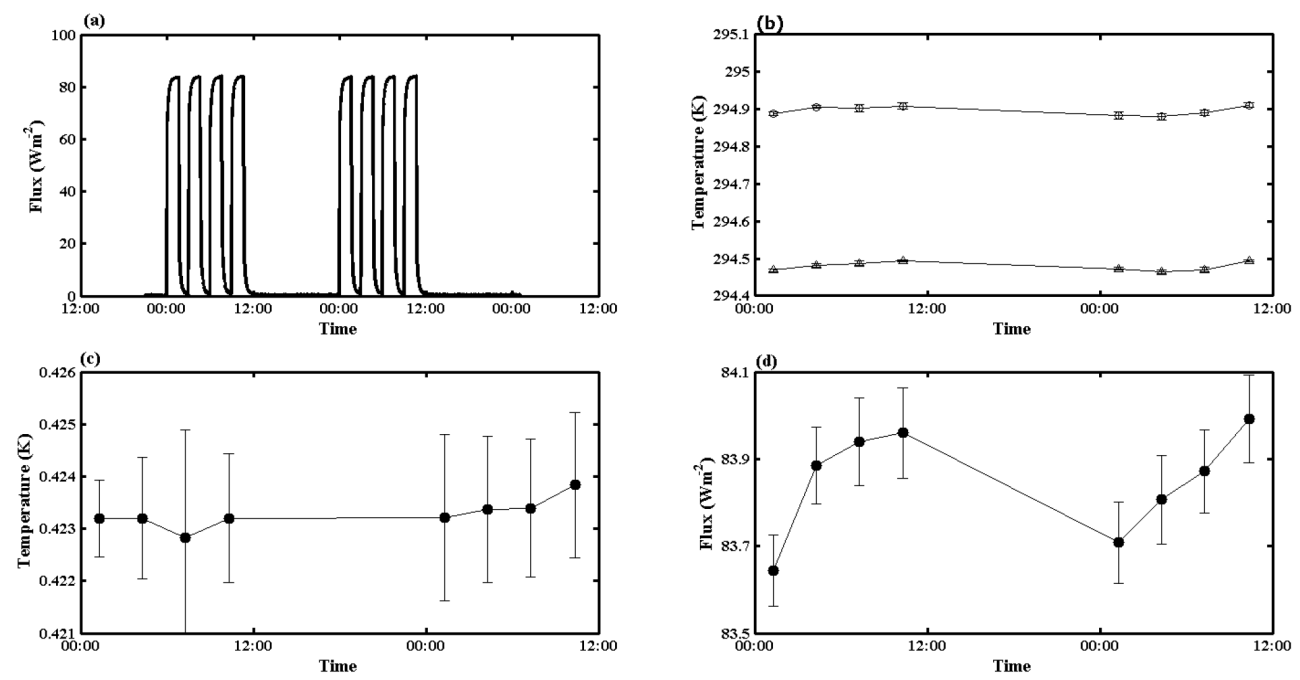

Figure 3. The instrument was tested in a temperature-controlled clean room. (a) Eight cycles of an on/off radiation source. (b) Temperatures of T1 and T2 measured around the peak of each cycle. (c) The temperature difference T1-T2. (d) Flux computed from temperatures T1 and $\mathrm{T} 2$.

it produces a very homogenous UV-VIS-NIR radiation. The radiation source generated by Labsphere arrived at the front transparent optical window of the vacuum tank after passing through a $1 \mathrm{~cm}$ aperture. The source is set to be automatically ON/OFF for eight measurement cycles. The duration of each measurement cycle is $99 \mathrm{~min}$, which is approximately the orbital period of PICARD (Fig. 3a).

The $T_{1}$ and $T_{2}$ temperatures were recorded with a period of $10 \mathrm{~s}$ for the eight repeated measurement cycles. The mean value and the standard deviation $(1 \sigma)$ of $T_{1}$ are $294.897 \pm 0.007 \mathrm{~K}, T_{2}$ is $294.480 \pm 0.004 \mathrm{~K}$, and the temperature difference $\left(T_{1}-T_{2}\right)$ is $0.423 \pm 0.001$ (Fig. $\left.3 b, c\right)$. The flux is calculated with Eq. (3) from the measurements of $T_{1}$ and $T_{2}$. The mean value and the standard deviation of the relative flux are $83.9 \pm 0.1 \mathrm{Wm}^{-2}$. The $1 \sigma$ value of temperature is at the level of milli-Kelvin, and that of the heat flux is about one-tenth of $\mathrm{Wm}^{-2}$ (Table 1). The signal shows a clear diurnal cycle variation in temperature and, more evidently, in flux (Fig. 3d).

\section{Observations from space}

The BOS sensor onboard the PICARD microsatellite was switched on on 28 June 2010. It has been continuously recording since then with a $10 \mathrm{~s}$ cadence. The total duty cycle of BOS observation is 1278 days. The complete space-acquired level 2 data set in $\mathrm{Wm}^{-2}$ is plotted in Fig. 4a. The global data set is categorised according to the physical origin of the signal (Fig. 4b). The complete full sampling data are published and accessible through the website: http://idoc-picard.ias.u-psud.fr/sitools/ client-user/Picard. The nominal mode represents the data when the sensor is only receiving the solar and terrestrial radiation at an altitude around $725 \mathrm{~km}$. During satellite occultations, the BOS sensor looks through the upper atmosphere to the Sun until its vision is completely blocked by the Earth. This happened every year between the beginning of November and mid-February.

MDO (Mode DistOrsion) represents the distortion mode of the satellite, during which the satellite was rotating clockwise along its Sun-pointing axis. MES (ModE Stellaire) is 

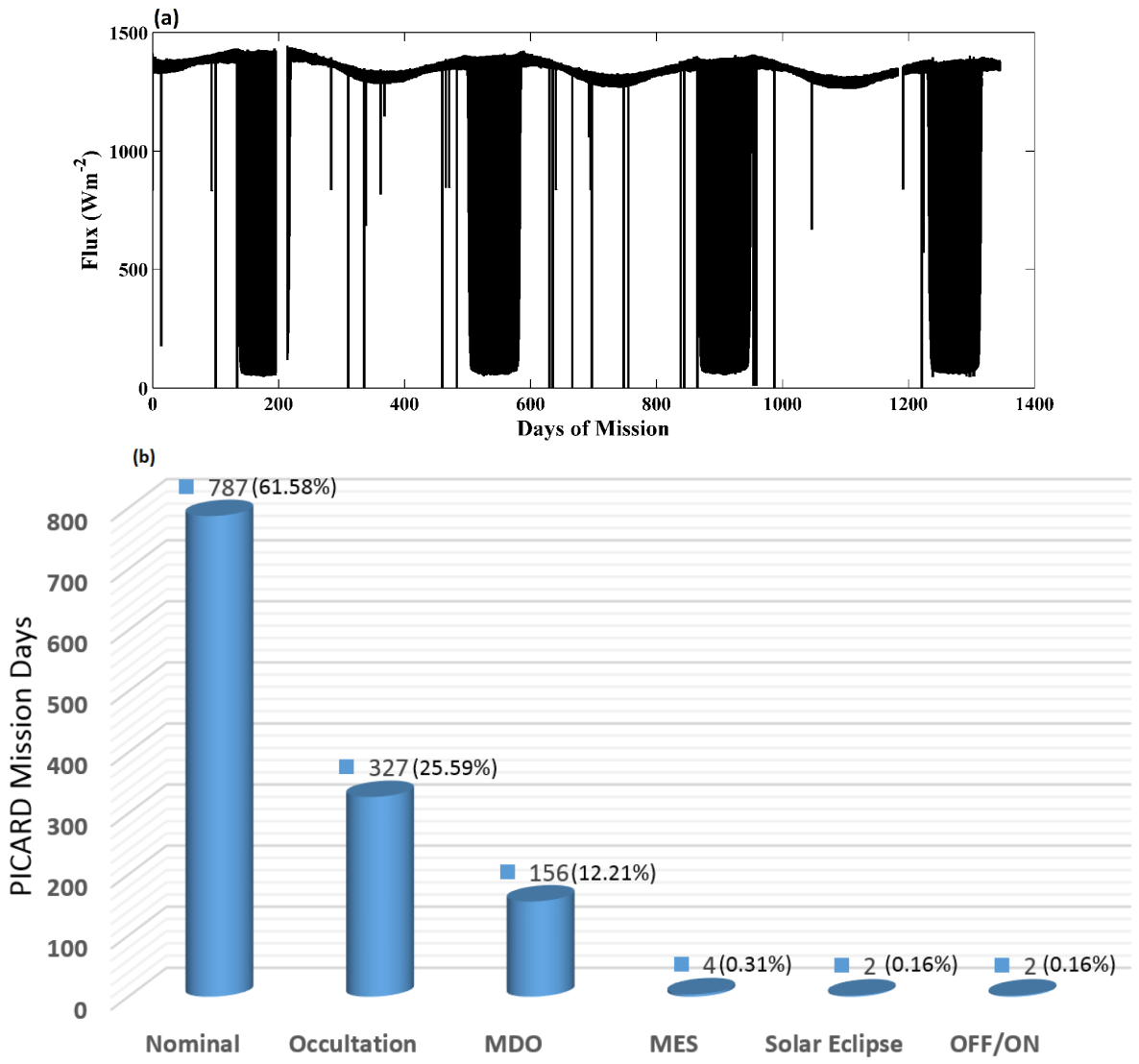

Figure 4. Observations during the entire PICARD mission. (a) Level 2 thermal flux. (b) Data partition according to its physical origin; unit is the day. Nominal: the measurements without any special operation. Occultation: observation during the satellite occultation by the Earth; it is related to the orbit of PICARD. MDO (MOde Distortion): the satellite is rotating along its Sun-pointing axis. MES (ModE Stellar): the Sun-pointing axis is turning away to a star. OFF/ON: the SOVAP experiment is switched off and on to test the electrical ground.

Table 2. MES calibration validation.

\begin{tabular}{lrrrrrrrr}
\hline Date & $T_{1}(\mathrm{~K})$ & $1 \sigma$ & $T_{2}(\mathrm{~K})$ & $1 \sigma$ & $T_{1}-T_{2}(\mathrm{~K})$ & $1 \sigma$ & $F\left(\mathrm{Wm}^{-2}\right)$ & $1 \sigma$ \\
\hline $2010 / 10 / 06$ & 299.4 & 0.13 & 301.4 & 0.13 & -2.226 & 0.0091 & 1411.9 & 0.65 \\
$2010 / 11 / 09$ & 301.8 & 0.13 & 303.6 & 0.13 & -2.256 & 0.0068 & 1388.0 & 0.43 \\
$2011 / 05 / 04$ & 302.7 & 0.09 & 304.8 & 0.12 & -2.286 & 0.0037 & 1389.5 & 0.77 \\
$2011 / 05 / 30$ & 302.4 & 0.19 & 304.5 & 0.11 & -2.278 & 0.0040 & 1370.9 & 4.70 \\
$2011 / 10 / 01$ & 305.4 & 0.14 & 307.5 & 0.14 & -2.379 & 0.0036 & 1371.5 & 0.86 \\
$2011 / 10 / 24$ & 305.9 & 0.12 & 308.1 & 0.13 & -2.381 & 0.0036 & 1378.6 & 0.49 \\
$2012 / 03 / 19$ & 306.5 & 0.13 & 308.7 & 0.13 & -2.388 & 0.0031 & 1372.0 & 1.19 \\
$2012 / 03 / 24$ & 306.4 & 0.13 & 308.6 & 0.13 & -2.386 & 0.0037 & 1371.5 & 2.59 \\
$2012 / 04 / 24$ & 305.7 & 0.13 & 307.8 & 0.14 & -2.362 & 0.0080 & 1365.2 & 3.62 \\
$2012 / 05 / 25$ & 304.4 & 0.10 & 306.5 & 0.13 & -2.323 & 0.0023 & 1369.7 & 1.88 \\
$2012 / 07 / 15$ & 304.5 & 0.13 & 306.6 & 0.13 & -2.318 & 0.0103 & 1351.7 & 5.15 \\
$2012 / 07 / 22$ & 304.7 & 0.13 & 306.8 & 0.13 & -2.331 & 0.0077 & 1355.2 & 2.57 \\
$2012 / 10 / 13$ & 307.2 & 0.13 & 309.4 & 0.13 & -2.419 & 0.0078 & 1361.8 & 1.04 \\
$2012 / 10 / 19$ & 307.4 & 0.13 & 309.6 & 0.13 & -2.419 & 0.0087 & 1368.7 & 0.83 \\
$2012 / 11 / 09$ & 308.1 & 0.15 & 310.1 & 0.15 & -2.422 & 0.0068 & 1343.7 & 0.30 \\
$2013 / 03 / 11$ & 308.4 & 0.13 & 310.5 & 0.14 & -2.424 & 0.0096 & 1360.4 & 0.91 \\
$2013 / 10 / 31$ & 308.6 & 0.12 & 310.8 & 0.13 & -2.444 & 0.0037 & 1354.7 & 0.71 \\
\hline
\end{tabular}



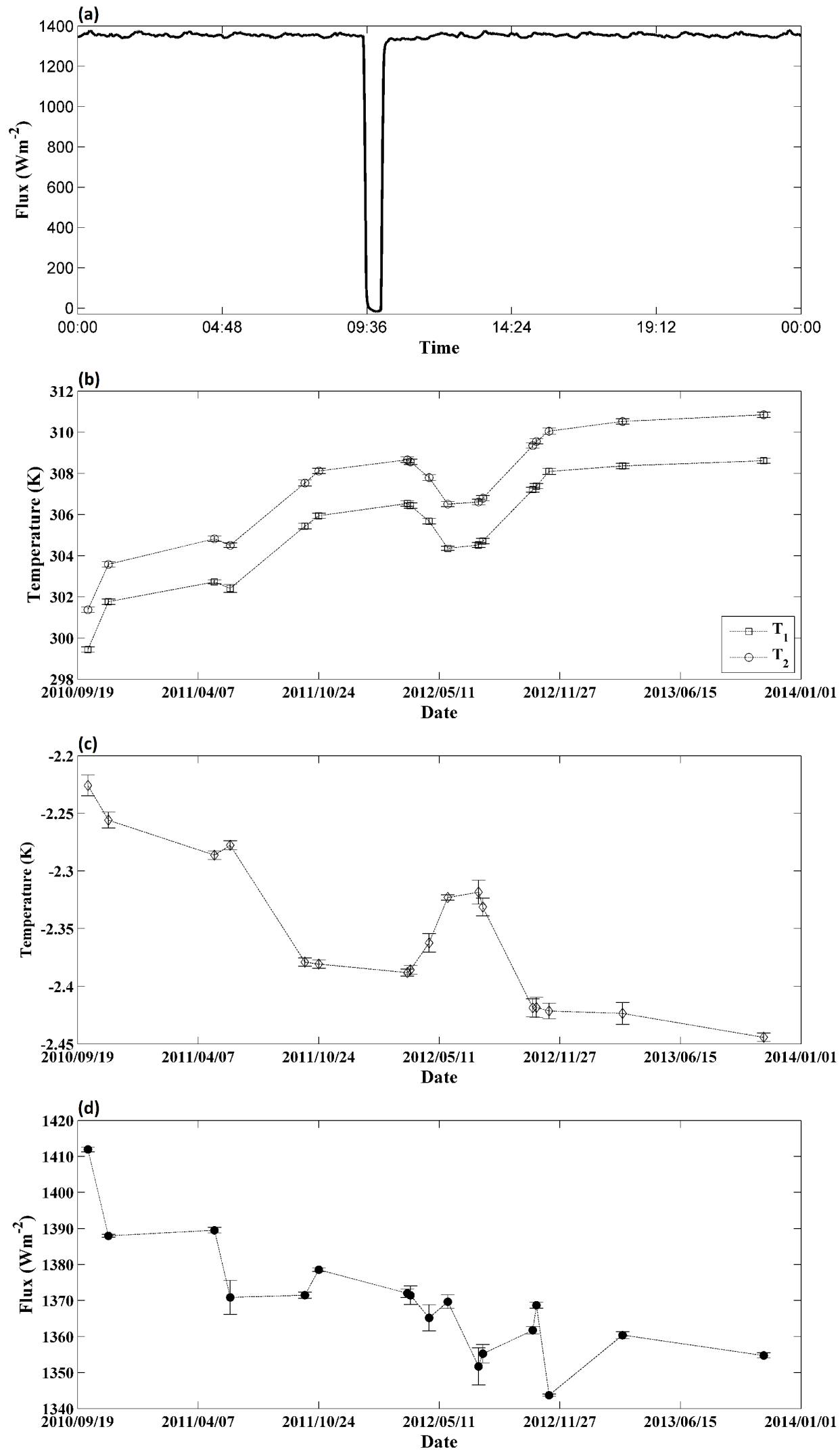

Figure 5. Star-pointing events (MES) observed in space. (a) One example of MES events. (b) $T_{1}$ and $T_{2}$ of all MES. (c) The temperature difference $\left(T_{1}-T_{2}\right)$. (d) The calibrated flux; it has been normalised to $1 \mathrm{AU}$. 


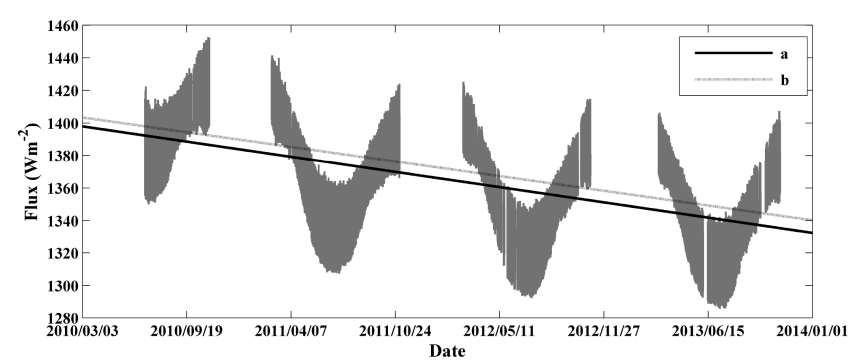

Figure 6. Decrease in the instrument sensitivity as obtained from the global data set (a) and from MES (b).

the star-pointing mode, in which the sensing unit was pointing to a star in deep space. The observations during MES are important for the calibration of all payload instruments. During the entire PICARD lifetime, there have in total been 22 MES operations. We consider 17 of them for the in-flight calibration of the BOS, excluding those with an abnormal positive minimum, observed inside the occultation period of 2013. The cause of the anomaly is still under investigation.

Contrarily to the laboratory experiment, the temperature is measured around the minimum value during MES (Fig. 5a), because it is like an OFF/ON of a radiation source and the laboratory experiment is an ON/OFF process of light. When the MES was started, the sensor passed a process.

During the MES, at the beginning, the sensor is still receiving solar energy. When the satellite is turning away from the Sun to a star, the energy is gradually decreased until it does not receive solar energy at all. The small valley around June to July in the records of $T_{1}$ and $T_{2}$ (Fig. 5b) and the corresponding plateau presented in Fig. $5 \mathrm{c}$ are due to the variation of solar and terrestrial radiation modulated by the Sun-Earth orbit distance change. Such effects are reduced by normalising the calibrated flux in a unit distance $1 \mathrm{AU}$ and correct velocity effects (Fig. 5d).

From 17 MES events, the mean value and the standard deviation of $T_{1}$ are $305.3 \pm 0.13, T_{2} 307.4 \pm 0.13 \mathrm{~K}$ and, $\left(T_{1}-T_{2}\right),-2.356 \pm 0.006 \mathrm{~K}$; the value of the calibrated flux $F$ is $1369.7 \pm 1.68 \mathrm{Wm}^{-2}$ (Table 2). The uncertainties of $T_{1}$ and $T_{2}$ are 2 orders and the flux $F 1$ order higher than during the laboratory experiment. Only the temperature difference $T_{1}-T_{2}$ shows a similar level error budget to the results of the laboratory experiment (Table 1).

The variation of the flux received by the BOS was modulated by the Sun-satellite distance which can be corrected using the knowledge of the position and attitude of the satellite. The BOS with its hemispherical field of view has been receiving both solar and terrestrial radiation above the top of the atmosphere (TOA), which can not be separated easily. After normalising the flux of MES to $1 \mathrm{AU}$, the decreasing radiative flux with time can be associated with the change in instrument sensitivity (Fig. 5d). The variation of the flux with time is calculated both from MES and from the global
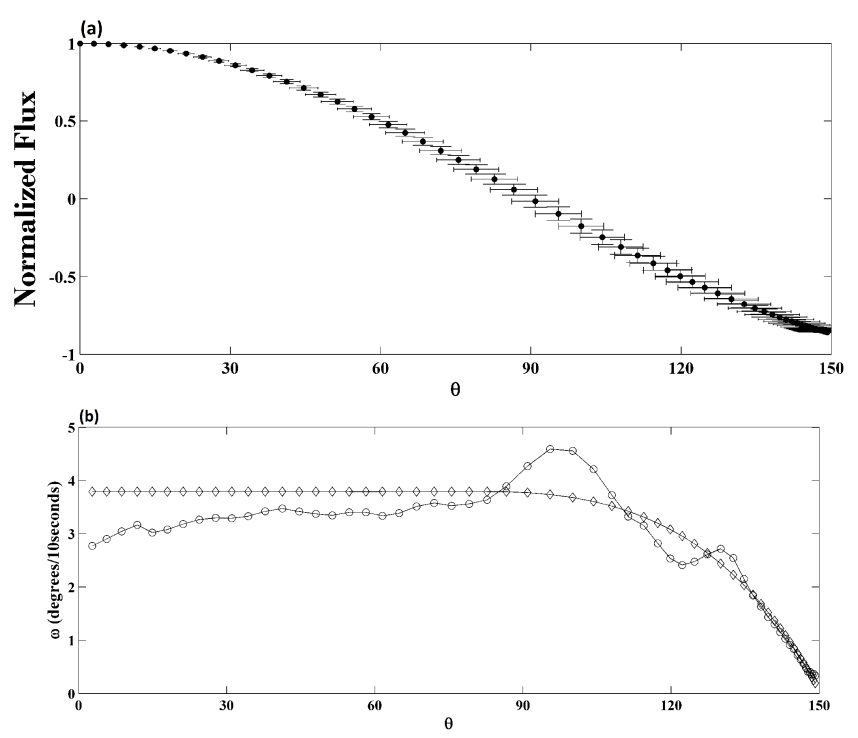

Figure 7. Upper panel: stacking results of 17 star-pointing (MES) observations during ascending phases. Lower pane: the diamond sign is the rotation of the spacecraft; the circle represents the motion inverted from BOS records.

data set. Its linear fits are represented in Fig. 6. The former analysis yields to a loss of $-16.5 \mathrm{Wm}^{-2} \mathrm{yr}^{-1}$ as the sensitivity decreases by $5 \%$ over the whole mission, while the latter suggests a loss rate of $-17.1 \mathrm{Wm}^{-2} \mathrm{yr}^{-1}$. Both results are in agreement within the $95 \%$ confidence level (Fig. 6).

\section{Lambertian performance}

The BOS has a hemispherical field of view (HFOV) and the detector is directly exposed to space without any optical or aperture accessories. In optics, Lambert's cosine law says that the radiant intensity or luminous intensity observed from an ideal diffusely reflecting surface or ideal diffuse radiator is directly proportional to the cosine of the $\theta$ angle between the direction of the incident light and the surface normal (Smith, 1966). The Lambertian performance is studied using the part of data obtained during MES, when the satellite is slowly turning away from the Sun-pointing axis to a star in deep space. We stacked all 17 MES records of descending phases; then, we removed the mean value and normalised the stacking results to unity (Fig. 7a).

A set of rotational angles was obtained by inverting the result with a cosine function. The first derivatives of angles $(\alpha)$ gave the angular velocities of the motion (degrees $10 \mathrm{~s}^{-1}$ ). If the detector has a cosine response to the incoming radiation, the angular velocity distribution should be close to the true rotation of the spacecraft. Since the angular motion of the spacecraft was precisely controlled by the Attitude Determination and Control (ADCS) of PICARD, the angular velocity of PICARD is known in advance. Two different sets of 

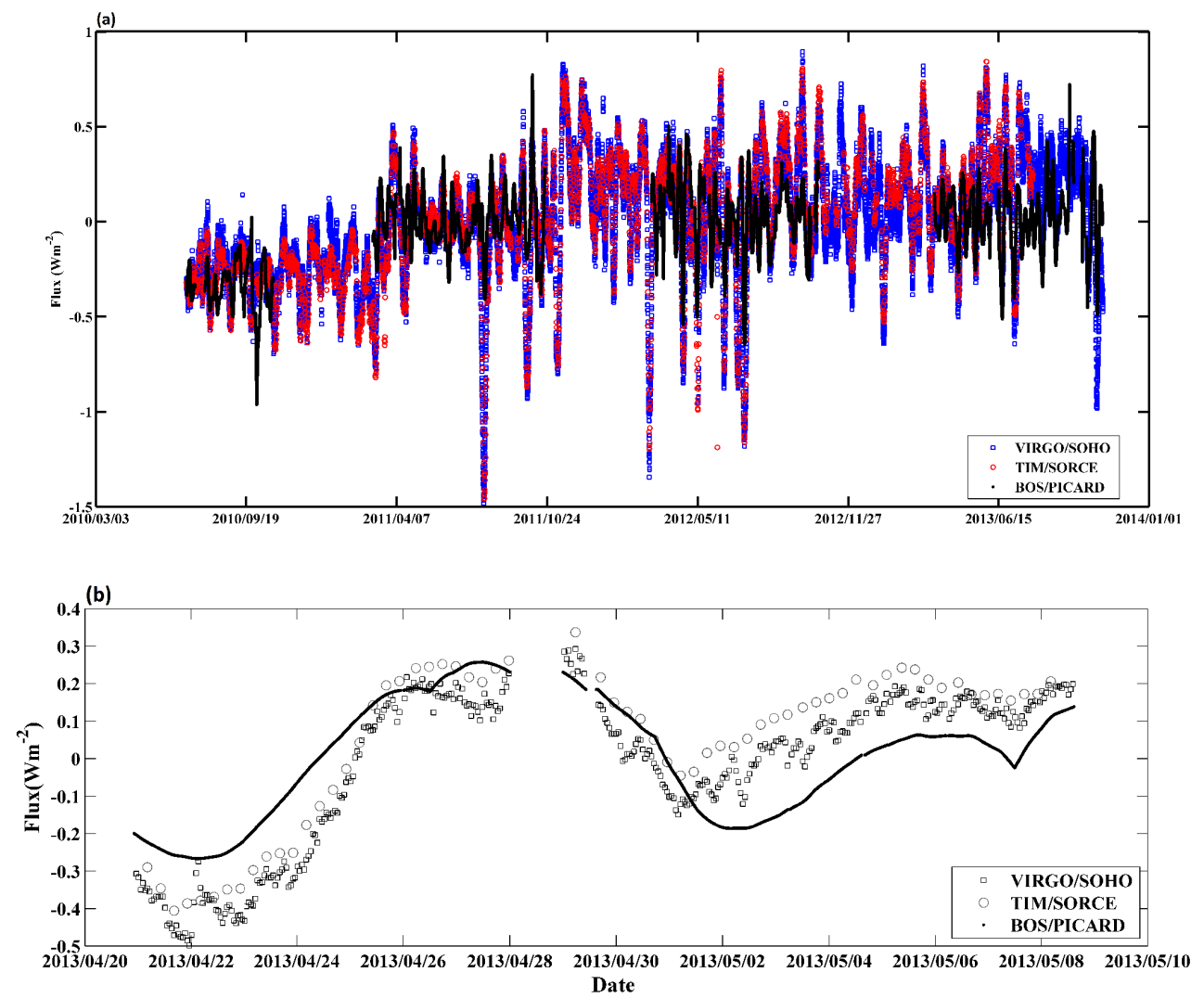

Figure 8. Comparison of the BOS with VIRGO/SOHO and TIM/SORCE. The upper panel shows the records during the whole PICARD lifetime. The cross-correlations are BOS-VIRGO 60.3\%, BOS-TIM 61.7\%, and VIRGO-TIM 97.5\%. The lower panel shows a zoom-in of the upper panel; the three measurements are plotted together with the sampling periods of $10 \mathrm{~s}$ for the BOS, $1 \mathrm{~h}$ for VIRGO and $6 \mathrm{~h}$ for TIM. The cross-correlation coefficients over this period are BOS-VIRGO 66.5\%, BOS-TIM $62.3 \%$, and VIRGO-TIM 90.5\%.

angular velocity are plotted together in Fig. 7b. The angular velocity from the BOS follows the true motion of the satellite well. The cross-correlation coefficient between them is $99.2 \%$. Thus, the instrument has a Lambertian response to the incoming radiation. As a consequence, the total radiation emitted by the Sun is equal to measured flux multiplied by the cosine of the pointing angle. In the case of PICARD, the pointing accuracy is better than $0.1^{\circ}$ (Meftah et al., 2014b). For the radiation of the Earth's origin, a correction factor has to be applied according to Lambert's cosine law.

\section{Preliminary comparison with absolute radiometers}

The variation of TSI is obtained after normalising to $1 \mathrm{AU}$, correcting orbital velocity effects (Frohlich et al., 1997) and removing the terrestrial radiation by a 7 day running mean. Since it is difficult to find a physical model of terrestrial radiation which could be directly applied to the records, we used the running average to remove the daily variation induced by terrestrial radiation. Several different window lengths have been tested; the one of 7 days is selected because it yields stable results for the solar radiation and terrestrial radiation.
For the solar radiation, we judge the results by comparing it with the satellite-Sun distance modulation. The terrestrial radiation is then projected onto a $1^{\circ}$ by $1^{\circ}$ map to be compared with the CERES product. The details about these higher-level products will be discussed in detail in the future works.

We compared the variation of TSI with data of VIRGO/SOHO (Frohlich et al., 1997) and TIM/SORCE (Kopp et al., 2011) over the same period. TIM/SORCE flies at a LEO (low Earth orbit) at an altitude of $645 \mathrm{~km}$ with an inclination of $40^{\circ}$. PICARD orbits the Earth at an SSO (sunsynchronous orbit) at an altitude of $725 \mathrm{~km}$, and the inclination is $98^{\circ}$. For radiometers onboard SORCE and PICARD, it receives the reflected visible and thermal radiation from the Earth, but since the absolute radiometer's main detector (black body) is in general installed at the bottom of a cavity, the terrestrial radiation contributions are maximally reduced. In addition, all cavities have a door to be operated in a sequence of opening and closing; the exposure time of the absolute radiometer is at least $50 \%$ shorter than PICARDBOS. For the computation of the TSI, we have not included the data observed during the occultation period, because of 
the influence of the terrestrial radiation on the main detector of the BOS.

VIRGO is a TSI composite from two absolute radiometers: the PMO6V and DIARAD from SOHO. The TIM/SORCE is another total solar irradiance measurement, which provides a new lower value of the solar constant. The variation of TSI is computed after removing the mean value of each individual data set. The sampling rate of VIRGO and TIM is linearly interpolated to $10 \mathrm{~s}$ to match the cadence of the BOS. Figure 8 shows the short-term variation of total solar irradiance from the three independent experiments. The crosscorrelation between VIRGO and TIM is $97.5 \%$. The value for VIRGO-BOS is $60.3 \%$, and TIM-BOS is $62.3 \%$. The BOS follows fairly well the TSI evolution of VIRGO/SOHO and TIM/SORCE, assessing the ability of the BOS to measure the TSI. The contribution of the terrestrial radiation, on the other hand, is more difficult to estimate due to the variation with time of the angle between the source and the sensor.

\section{Conclusions and discussions}

The BOS has worked in space for 3.5 years. The sensibility of the absolute temperature measurements has been determined in the laboratory to be of the order of $10^{-3}$. In-flight data rather suggest uncertainties of the order of $0.1 \mathrm{~K}$, which is 2 orders higher than the laboratory results. The $1 \sigma$ value of flux determination is $1.6 \mathrm{Wm}^{-2}$; it is about 10 times that of laboratory results. The temperature of $T_{1}$ and $T_{2}$ was increased from 306 to $326 \mathrm{~K}$ and 301 to $322 \mathrm{~K}$ respectively since the beginning of the mission. The $20 \mathrm{~K}$ temperature rise was not sufficient to explain the disagreement in the error budget between the space observations and the laboratory experiment.

The uncertainty in the short-term solar irradiance variation is $\pm 0.45 \mathrm{Wm}^{-2}$ after removing the terrestrial radiation (Zhu et al., 2014). It hints at the fact that the larger error bar deduced from MES could be due to the contribution of terrestrial radiation that was permanently recorded by the BOS during the entire mission. It proves that we are able to improve the relative uncertainty but obviously not the absolute accuracy. On the other hand, the errors in the temperature difference measurements $\left(T_{1}-T_{2}\right)$ are in milli-Kelvin, calculated from both laboratory and space experiments. It shows that the ECM and ambient thermal noise are efficiently reduced with the design of electronic interface and the thermal structure of the BOS instrument.

Globally, the BOS shows a linear degradation of $-16.8 \pm 0.4 \mathrm{Wm}^{-2} \mathrm{yr}^{-1}\left(-33.7 \pm 0.8 \mathrm{ppm} \mathrm{day}^{-1}\right.$ to the TSI amount). It is 1 order higher than the degradation of the PMO6V and DIARAD absolute radiometers onboard the SOHO satellite (Frohlich et al., 1997). The high degradation rate could be linked to the longer exposure time. A robust relation between the exposure time and the detector's degra-
Table 3. Instrument data sheet: * diameter; ${ }^{* *}$ sensibility.

\begin{tabular}{lr}
\hline Parameter & Data \\
\hline Mass $(\mathrm{g})$ & 168 \\
Volume $\left(\mathrm{cm}^{3}\right)$ & 366.7 \\
Height $(\mathrm{cm})$ & 9.13 \\
${\text { White surface }(\mathrm{cm})^{*}}^{*}$ & 6.34 \\
Black surface $(\mathrm{cm})^{*}$ & 4.65 \\
Shunt $(\mathrm{cm})^{*}$ & 1.81 \\
Absolute temperature $(\mathrm{K})^{* *}$ & $10^{-1}$ \\
Relative temperature $(\mathrm{K})^{* *}$ & $10^{-3}$ \\
Flux $\left.(\mathrm{Wm})^{-2}\right)^{* *}$ & $10^{0}$ \\
Operating temperature $\left({ }^{\circ} \mathrm{C}\right)$ & {$[-30,+60]$} \\
Power consumption $(\mathrm{mW})$ & {$[100,300]$} \\
\hline
\end{tabular}

dation could be established when the UV dose can be simultaneously monitored.

The instrument-sensitive mode of the absolute radiometer is better modelled with a hyperbolic function. However, from the current space cumulated data, the linear regression function is the best fit for the change in BOS sensitivity. Taking into account that the main detector either of the BOS or the absolute radiometer is black coated, it suggests that the nonlinearity of the absolute radiometer's degradation is mainly induced by the optical part rather than by the painting, a phenomenon which is designated as the "siliconising" of the quartz window exposed to UV radiation (Frohlich, 2003).

The BOS is a perfect Lambertian detector. A cosine correction must be applied to the total amount of radiation. The contribution is small since the pointing angle is stable in the Sun's direction. The correction is more important for the terrestrial radiation, where the pointing angle between the source and the sensor is bigger.

The BOS is a TRL level-9 space instrument after its successful service to the PICARD mission according to the definition of ESA's technology readiness level (ESA, 2012). The details of the instrument are listed in Table 3. The space performance of the instrument proves that the BOS is able to measure a large dynamic range of electromagnetic radiation with a relative high resolution. The electronic and thermal design shows its high efficiency in reducing the ECM and ambient thermal noise. The BOS is a stable sensor to track the small amount of variation in the electromagnetic radiation. It can be payloaded on the micro, even CubeSat, missions to study the irradiance from the solar as well as planetary origin.

Acknowledgements. We appreciate the discussion on the ground experiment given by referee M. Reina. The comments given by the anonymous reviewers have helped us to improve the quality of the paper. The PICARD mission is supported by Centre National d'Etudes Spatiales (CNES), the CNRS / INSU, the Belgian Space Policy (BELSPO), the Swiss Space Office (SSO), and the European Space Agency (ESA). We would like to thank Ali Ben Moussa 
and B. Giordanengo from DeMeLab, ROB (Royal Observatory of Belgium), who provided us with the Labsphere and source calibration, and Marie Dominique for her valuable comments on the manuscript. F. Clette gave valuable suggestions for data analysis. J.-P. De Cuyper welcomed us to do the experiment at his digital laboratory. The BOS experiment is financed by the Belgian PRODEX program, and the ESA project with the management support of BELSPO.

Edited by: M. Díaz-Michelena

\section{References}

Dewitte, S., Crommelynck, D., Mekaoui, S., and Joukoff, A.: Measurement and uncertainty of the long-term total solar irradiance trend, Solar Phys., 224, 209-216, 2004.

ESA: Strategic readiness level, available at: http://sci.esa.int/sre-ft/ 37710-strategic-readiness-level/ (last access: 7 May 2015), 2012.

Fröhlich, C.: Long-term behaviour of space radiometers, Metrologia, 40, S60, doi:10.1088/0026-1394/40/1/314, 2003.

Fröhlich, C., Crommelynck, D. A., Wehrli, C., Anklin, M., Dewitte, S., Fichot, A., Finsterle, W., Jiménez, A., Chevalier, A., and Roth, H.: In-flight performance of the Virgo solar irradiance instruments on SOHO, Solar Phys., 175, 267-286, doi:10.1023/A:1004929108864, 1997.

K \& K Associate: Thermal network modeling handbook, Version 97.003, available at: http://www.tak2000.com (last access: 7 May 2015), 2000.

Kopp, G. and Lean, J. L.: A new, lower value of total solar irradiance: evidence and climate significance, Geophys. Res. Lett., 38, 1706, doi:10.1029/2010GL045777, 2011.

Labsphere: Uniform source spheres (PB-13019-000), available at: http://www.labsphere.com (last access: 7 May 2015), 2005.

Martinez, L. D.: Participation à la conception et à la réalisation du capteur BOS destiné à mesurer la variabilité de l'irradiance solaire totale, thesis, Annee Academique, 2006-2007, Université Libre de Bruxelles, 2007.

Measurement specialties: Mini BetaCURVE Thermistor Probe (MDB), available at: http://www.meas-spec.com (last access: 7 May 2015), 2005.

Mekaoui, S., Dewitte, S., Conscience, C., and Chevalier, A.: Total solar irradiance absolute level from DIARAD/SOVIM on the International Space Station, Adv. Space Res., 45, 1393-1406, 2010.

Meftah, M., Hochedez, J.-F., Irbah, A., Hauchecorne, A., Boumier, P., Corbard T., Turck-Chièze, S., Assus, P., Bertran, E., Bourget, P., Buisson, F., Chaigneau, M., Damé, L., Djafer, D., Dufour, C., Etcheto, P., Ferrero, P., Hersé, M., Marcovici, J.-P., Meissonnier, M., Morand, F., Poiet, G., Prado, J.-Y., Renaud, C., Rouanet, N., Rouzé, M., Salabert, D., and Vieau, A.-J.: PICARD SODISM, a space telescope to study the Sun from the middle ultraviolet to the near infrared, Solar Phys., 289, 1043-1076, 2014a.
Meftah, M., Dewitte, S., Irbah, A., Chevalier, A., Conscience, C., Crommelynck, D., Janssen, E., and Mekaoui, S.: SOVAP/Picard, a spaceborne Radiometer to Measure the Total Solar Irradiance, Solar Phys., 289, 1885-1899, 2014b.

Miller, R.: DuPont Engineering Technical Information, Polymeres, Vespel polyimide parts and shapes, available at: http://www. dupont.com (last access: 7 May 2015), 2000.

Schmutz, W., Fehlmann, A., Finsterle, W., Kopp, G. and Thuillier, G.: Total solar irradiance measurements with PREMOS/PICARD, AIP Conf. Proc., 1531, 844, doi:10.1063/1.4804902, 2013.

Smith, W. J.: Modern optical engineering, Tata McGraw-Hill Education, p. 228, 1966.

Thullier, G., Dewitte, S., Schmutz, W., and The Picard team: Simultaneous measurement of the total solar irradiance and solar diameter by the PICARD mission, Adv. Space Res., 38, 17921806, doi:10.1016/j.asr.2006.04.034, 2006.

van Ruymbeke, F.: Mise au point d'un capteur embarqué sur satellite destiné à mesurer les variations du flux radiative solaire, thesis, Annee Academique 2005-2006, Université Catholique de Louvain, 2006.

van Ruymbeke, M., Renders, F., Noël, J.-P., van Ruymbeke, F., Martinez, D. L., and Zhu, P.: Mise en orbite d'un bolomèter élaboré à l'Observatoire Royal de Belgique 1ère Partie: Description du dispositif. Ciel et Terre, 126, 173-179, 2010.

van Ruymbeke, M., Zhu P., Renders, F., and Noël J.-P.: Mise en orbite d'un bolomèter élaboré à l'Observatoire Royal de Belgique 2ème partie : Présentation d'observations de divers phénomènes enregistrés en orbite par le BOS, Ciel et Terre, 127, 14-16, 2011.

Zhu, P., Ruymbeke, M. V., Meftah, M., Clette, F., Dewitte, S., Chevalier, A., van Ruymbeke, F., and Noel, J.: The preliminary measurements from the Bolometer Oscillation System (BOS) on board PICARD, American Geophysical Union, Fall Meeting 2010, abstract \#GC21B-0873, 2010.

Zhu, P., Karatekin, Ö., Noël, J.-P., van Ruymbeke, M., and Dehant, V.: EGU General Assembly 2012, 22-27 April 2012, Vienna, Austria, p. 7271, 2012.

Zhu, P., Karatekin, Ö., van Ruymbeke, M., Dewitte, S., and Thuillier, G.: The solar irradiance registered at a flat-hemispherical field of view-bolometric oscillation sensor on board PICARD satellite. EGU2014-5112, EGU General Assembly, 27 April-2 May 2014, Vienna, Austria, 2014. 